\title{
Design and Experimental Study of Automatic Delivery Device for Granular Material Based on PLC
}

\author{
Wang Yongdong \\ Liaoning Jianzhu Vocational University, Liaoyang, Liaoning111000, China
}

Keywords: poultry breeding; pellet feeding; automatic delivery; PLC

\begin{abstract}
Along with the development and progress of the society, the poultry industry in China is gradually to the level of modernization development, in order to realize the automation of poultry breeding in accurate feeding, with the investigation and analysis of effectiveness, this paper has designed a kind of automatic feeding device of pellet feed based on PLC. The device is the main power control core with PLC machine, based on position detection device with proximity switch, and electromagnet drive blanking parts by manual action on the touch screen is set for the blanking time, in order to achieve quantitative automatic feeding [1].
\end{abstract}

\section{Introduction}

Due to the continuous improvement of science and technology level, poultry farming in China has some problems in the past traditional poultry farming mode, which requires a lot of manpower and material resources to be wasted. At present, China has begun automatic feeding in poultry scale farming, and the level of automation is improving. It realizes the precise feeding in poultry feeding, to a certain extent, illustrates the demand of poultry breeding industry for automatic feeding. At present, in the field of poultry breeding in the world, it is commonly used in the field of poultry breeding and has a better way to develop the feeding mode of "one egg breeder". Next, we will take "one egg breeder chicken" as an example to illustrate that the breeding of "one egg breeder chicken" is mainly based on a laminated feeding way, feeding poultry animals in an automated way. There is a great advantage over the feeding efficiency, the labor force and the traditional way. In recent years, China has basically implemented feeding on cattle and sheep in animal husbandry, and its research on automatic feeding device has achieved better results. Most of the automatic feeding devices used are walking. But less research on automatic feeding links in poultry categories in the present stage of our country, and because poultry feeding and poultry rearing methods have some differences, so in the feeding time, study on the scientific and reasonable feed type and feed ratio should be, for feeding in poultry feed automatic dispensing device can not be directly applicable to the birds, so this will be for the birds feed automatic dispensing device is studied, has a very important significance [2].

People in order to more effectively reduce the design and production cost of the automatic delivery device, and improve the reliability of the automatic delivery system more effectively, we design a device with automatic blanking function, which can feed poultry feed. Such a device system is installed in a feeding device in most cases. It ensures that when feeding poultry, it can carry out ration feed according to the way of walking feeding, and then complete a complete feeding process for poultry. So, scientific and reasonable design of poultry feed delivery device should be carried out experimental study on blanking weight on the relationship between grain size degree of automation device blanking blanking device, and the weight of feed and feeding the time, provides a good theoretical basis for the research of walking system feeding device.

\section{Mechanical Structure and Work Situation in Automatic Delivery Device of Granular Material}

At the present stage, the equipment used in poultry breeding in China is often applied to the following parts when feeding the feed. The hopper, the upper plate of the material mouth, the 
middle layer plate and the blanking tube are made up. The feeding port of the device is mainly composed of three important parts, upper, middle and lower, and is made up of the laminate of the blanking mouth and the plugged plate of the material mouth. In order to ensure the material sliding action better able to make a plate inserted in the mouth repeatedly, to ensure that the outlet plate thickness is less than the discharge port version of the middle thickness, such a device in discharge port and discharge port inserted in the upper plate are stainless steel sheet with thickness less than $1 \mathrm{~mm}$, which can effectively prevent falling material blocking situation the material in the process of. The electromagnet in both ends of the automatic delivery device should be installed in the opposite direction, so that the magnet can be used as a driving force element for the device to work, so as to promote the opening and closing actions of the feed gate. Because the inserts in the device can also slide up and down, the feed can be fed to a certain extent according to the different size of the feed [3].

In view of the design of the automatic delivery device for poultry, the device is at the beginning at the beginning of the rest of the outlet. When the automatic delivery device for the work, when positioning patch can achieve device switch position, it will produce a high level signal device, and effectively transmitted to the PLC, then PLC can effectively control the operation in a short period of time to ensure that the electromagnet, electromagnet device can be inserted in the movement the outlet of case driven, fall to complete the opening and closing action of feed. When the device is set in the blanking movement, automatic delivery device PCL control will be even electromagnet with the faster speed of electric equipment, to a certain extent, promote the automatic equipment delivery orifice plate movement in the opposite direction, to complete the automatic feeding device in a full set of action. After the series of actions are completed, the PCL in the device will accept the information of power and turn on and off the device. The touch screen in the device can be applied to input a certain drop time so that all automatic feeding devices can be realized [4].

\section{Control System in the Automatic Delivery Device of Granular Material}

In the automatic feeding device for granular materials, the control system principle is rather complex. The PLC in the control system is SIEMENSS7-200 type, and the CPU in the device is 222 type, and the voltage of 24 volts is used for power supply. The touch screen in the device and the PLC have a fixed communication mode. Inductive proximity switches generally use TCA-3050A type, and the working voltage is 6-36 volts, the output is NPN open type, the detection distance of metal objects is generally $0-55 \mathrm{~mm}$; the general relay uses the RJ2S-CL-D24 model, the fixed coil rated voltage is 24 volts; characteristics of electromagnet device should be chosen in the the 24 volt DC push-pull electromagnet, the electromagnet drive current is 6 amperes, putting stroke which is $34 \mathrm{~mm}$, with the thrust of about 20 Newton, can be more effective for the conduction time in the device rules within 2 seconds.

Aiming at the problem of driving load capacity in many automatic delivery devices, a resistance with a certain resistance can be connected between the signal terminal of the delivery device and the positive pole of the power supply. When the feed is automatically put in, when the switch of the power supply is induced to the metal object, the PLC in the device will appear a certain high level signal. In the PLC program, set its intermediate state register effective, can be used to eliminate jitter and proximity switch sensing, is capable of handling signals generated in the process out of the electromagnet, can reduce a circuit breaker in the circuit damage of electromagnet in automatic equipment. In the device program to achieve further work, that is, each time only need and require a set of relays to control the electromagnet.

\section{Test Results and Analysis}

The object of this test is put in the raw food pellet feed mixture were studied. Test results show that: in the outlet opening area fall automatic delivery device is always unchanged, the average weight of material falling device and blanking time shows certain linear relationship, to a certain extent research automatic feeding device for poultry research variables provides a theoretical basis 
and research direction of good [5].

In order to simulate the real situation of feed feed for poultry feed more effectively, this study chooses feed made from raw grain mixed together, such as millet, sorghum, millet and corn. In the process of experiment, we can input the blanking time on the touch screen of the device, distinguish it according to the time and length, and then click the blanking button to complete a complete feed delivery process. Moreover, the experimenter can use the electronic balance to accurately weigh and record the feed every time, so as to analyze the relationship between the importance and delivery of feed. The injection and weighing will be carried out 100 times for the drop weighing test under different blanking time conditions, and the feed data will be finally obtained [6].

When the opening area of the opening in the device is constant, the tester should take different blanking time as the variable to measure the result of different test. The tester can also use the drop material as the variable to test, and finally get the different weighing results. The results show that the size of the material and the drop time of the device are regularly adjusted, and the amount of the drop material in the test is different. In the experiment, a single influence factor did not happen, so the weight of the blanking material increased gradually with the increase of blanking time and the area of the blanking orifice. If the opening area of the blanking remains the same, the variance between the data displayed during the test will be very large. Effective analysis of blanking error for the following reasons. First, choose the magnet device is short, the driving force is relatively small, once the chutes appear closed, then it will cause the feed device plugging in the outlet position, so that the low efficiency of feeding failure. It will also lead to the emergence of big grain feed when it is closed, and it will be blocked in the gap which has not been closed. It will cause great influence on the weighing and calculation of the next blanking data. According to the test results can be obtained in different outlet, device area and blanking time and blanking weight has a linear relation with the increase of test variables, dropping time, so the test results of the blanking average weight showed a linear increase. With the increase of the opening area of the test variable, the time of the blanking and the average weight of the blanking increase linearly.

\section{Conclusion}

Research on automatic dispensing device design and particles of PLC can be seen, if the device in the blanking port opening area as a constant, then the test variables in blanking blanking, the average weight of time will show a linear correlation. When the correlation between the blanking time and the blanking weight is calculated, the slope of the test data will change gradually with the change of the opening area of the blanking orifice.

For use in test of the feed delivery material is raw food mixed feed selection research, so the test will be analyzed for feed chutes often blocked phenomenon, to ensure the device error of the feed will be put to a minimum, and then carry on the analysis and Research on effective, ultimately developed the feeding device is more convenient and convenient. Besides, after that, the scientific and reasonable selection of the power components inside the device can be done, and the structural foundation of the blanking parts can be analyzed and studied, so that the variance of the blanking weight can be effectively reduced. It is convenient to carry out the stability of the internal operation after the automatic feeding device of the granular material, and to realize the automatic design of automatic delivery device for granular material.

Aiming at the application of automated production line with automatic reclaimer in the market at present stage, it has made great progress both in the process of the device and in the control scheme of the device, which has a good research significance. By use of the PCL control system, it can effectively achieve the small volume of the device, and the control is more flexible in the failure test is also more flexible, which can improve the ultimate goal of productivity to a certain extent, in order to improve the final poultry production project.

The design of automatic delivery device based on PLC has many advantages. First, the device control is relatively simple. The user can get the material through the machine through the one button button, and ultimately achieve the automatic control of the whole process of feeding and feeding. Second, the automatic particle delivery device can be embedded in a large and more 
stereoscopic process program, and can be used as a process. Third, the pellet automatic delivery device has a strong expansion. It can expand the register settings and hardware expansion in PLC to expand the system to adapt to more complex working environment. Fourth, can be delivered accurately and quickly. Using PCL and pulse output module can realize the idea, and make precise positioning for decoration, so that it can more effectively guarantee mechanical manufacturing and various processing problems.

Conclusion: in summary, with the development of the times and the improvement of the level of science and technology. At present our country when feeding on poultry has gradually started to take automatic feeding, but less research on automatic feeding device, if it can be more efficient feeding, how to improve the feeding efficiency, is the key point we study the following content. Due to the increase in poultry feeding and poultry rearing methods have some differences, so in the feeding time, the study of scientific and reasonable consumption of feed ratio and feed raw materials should be fed to poultry feed, the automatic dispensing device can not be directly applicable to poultry, so this paper aimed at the automatic poultry feed put on the device are analyzed, and has a very important significance of the study. Since people are more effective in reducing the construction cost and improving work efficiency of the feeding device, the device should be installed on the top feeding device to realize the quantitative delivery of the feeding mode and complete a complete automatic feeding process. Scientific and reasonable design of poultry feed delivery device should first test the weight of blanking, providing a good theoretical basis for subsequent research on walking feeding system. The object of the trial feeding is the grain feed mixed with the original grain. To some extent, the test results show that when the opening area of the automatic delivery device is always unchanged, the average weight of blanking material and the time of blanking will show a certain linear relationship. If the device in the blanking port opening area as a constant, then the test variables in blanking blanking, the average weight of time will show a linear correlation. When the correlation between the blanking time and the blanking weight is calculated, the slope of the test data will change gradually with the change of the opening area of the blanking orifice.

\section{References}

[1] Narang I, Bharota I K, Raisuddin S, et al. Design and Experimental Research of Automatic Control System of Material Transport Car Based on Siemens S7-300 PLC[J]. Applied Mechanics \& Materials, 2015, 703(1):277-280.

[2] Zhang Y, Shen J. Design and Research of Car Rear Axle Welding for Automatic Alignment Device Based on PLC[J]. Modern Manufacturing Technology \& Equipment, 2016.

[3] Zhang X, Ju H, Guo D, et al. Design of an auto-removing mechanism based on a PLC control system[C]// International Congress on Image and Signal Processing. IEEE, 2016:1293-1297.

[4] Wang J, Wang S, Chen J, et al. Research on Automatic Irrigation Algorithm of Strawberry Greenhouse Based on PLC[M]// Computer and Computing Technologies in Agriculture VII. Springer Berlin Heidelberg, 2013:41-51.

[5] Feng Q X, Tian Z B, Tang Y P, et al. Automatic material delivery device on a large type of Stamping machine based on the PLC control[J]. Forging \& Stamping Technology, 2006, 31(2):65-66.

[6] Wumaier Tuerxun, Mu H, Xia Q. Design of Greenhouse Monitor and Control Device for Automatic Prevent from Gale Based on PLC[J]. Journal of Agricultural Mechanization Research, 2015. 